\title{
MÈT KONTÈ SE MANTI ! MAÎTRE CONTEUR, TU MENS OU TU (TE) TROMPES ! LE PASSAGE DE LA SIMULATION NARRATIVE À LA CONFESSION AUTOBIOGRAPHIQUE DANS LE CONTE HAÏTIEN EN DIASPORA
}

\author{
Sara DEL Rossi
}

Université de Varsovie

\begin{abstract}
En): The aim of this paper is to analyse the permeable boundaries between the author and the narrator in the rewritings of Haitian traditional oral tales, in the context of the presentday Diaspora. We will focus in particular on two storytellers, Joujou Turenne (Québec) and Mimi Barthélémy (France), to investigate the passage from the oral to the written form of a tale, a passage that disrupts the tale's narration as far as the traditional forms of opening and closing, the ultimate site of the narrator's simulation, are concerned. Our analysis will be based on a comparative study of the two storytellers' texts, by way of the specific case of the Haitian storyteller Paula Clermont Péan.
\end{abstract}

Keywords (En): Haitian folktale ; Mimi Barthélémy ; Paula Clermont Péan ; Joujou Turenne ; simulation ; oral literature

Mots-clés (Fr): conte haïtien; Mimi Barthélémy; Paula Clermont Péan ; Joujou Turenne ; simulation ; oraliture

\section{Introduction}

En Haïti, l'expression Pawol la pale (littéralement : la parole a été dite) peut être interprétée de deux façons : soit pour mettre en évidence l'importance de la parole énoncée, dans le sens de donner la parole à quelqu'un, soit pour souligner la vérité de la chose affirmée en la renouant à la véridicité du passé. Ce dernier sens concerne proprement l'oraliture, terme forgé dans les années 1970 par Ernst Mirville $^{1}$ pour substituer l'expression «littérature orale », qui désigne tous les moyens de divulgation et transmission du savoir de génération en génération. L'oraliture haïtienne, c'est-à-dire tous les genres oraux qui possèdent une structure à textualité mémorielle, non seulement est très vaste, mais elle est surtout encore très pratiquée au point d'influencer la littérature écrite, comme le constate Maximilien LAROCHE (2000 : 2) :

En Haïti, il y a toujours eu cette utilisation par les écrivains de l'oraliture populaire. La littérature haïtienne, principalement écrite en français, est basée sur l'oraliture populaire, c'est à dire cette expérience du peuple haïtien énoncée dans des chants, des proverbes, des récits, des contes. Tout cela constitue l'oraliture populaire.

Parmi les genres de l'oraliture tels que les jédmo (devinette-énigme), les blag (histoires drôles) ${ }^{2}$ et les lodyans ${ }^{3}$ (récit satyrique sur la société et la politique), le conte, conçu comme récit oral fictif structuré, est peut-être le genre le plus connu.

\footnotetext{
${ }^{1}$ Voir LAROCHE (1991:15-16).

${ }^{2}$ Pour plus de renseignements sur les genres de l'oraliture haïtienne consulter TESSONNEAU (2002).

${ }^{3}$ Pour plus de renseignements sur la lodyans consulter ANGLADE (1999) ou LEVY (2004).
} 
En ouvrant n'importe quel ouvrage ou article ethnographique, le but préfixé du conte est toujours le même : amuser en éduquant. Comme le souligne Jacqueline THOMAS (in TsOUNGUI, $1986: 87$ ) :

Les coutumes, les croyances et les traditions se transmettent de siècle en siècle par le canal des contes et les jeunes générations assimilent ce contexte socioculturel, sans même s'en rendre compte [...] Le conte négro-africain ne se contente donc pas de distraire l'auditoire, mais il maintient au sein de la communauté tout ce qui en fait la civilisation propre et la distingue des communautés voisines.

De ce fait, le conte permet d'acquérir les grandes vérités, les notions et les savoirs qui forment la culture d'un peuple, ses fondements. À l'intérieur d'un simple récit fictif, s'entremêlent des valeurs traditionnelles théoriques et pratiques (de la rhétorique à la maçonnerie) et les aventures fictives des héros.

Pourtant le conte, même en tant que source et diffuseur de la vérité de la tradition, ne peut pas être considéré comme un genre figé, puisqu'une de ses caractéristiques principales est la variation, c'est-à-dire la capacité de s'adapter toujours au contexte social en retenant les structures et les thèmes narratifs traditionnels. Cette manière de se renouveler à chaque fois, d'être au pas avec la société, caractérise les contes haïtiens contemporains, surtout en ce qui concerne l'appropriation du récit de la part du conteur, qui le maîtrise afin d'en donner sa propre version, enrichie par ses expériences personnelles et son contexte social particulier.

Cette étude se propose d'analyser les frontières perméables entre l'auteur et le narrateur dans les réécritures des contes oraux de la tradition haïtienne dans le contexte diasporique contemporain, plus précisément dans les cas de Joujou Turenne au Québec et de Mimi Barthélémy en France, en utilisant l'œuvre de la conteuse haïtienne Paula Clermont Péan en tant que terme de comparaison. En particulier, il s'agira d'examiner comment le conte écrit contemporain en contexte diasporique n'est plus seulement un véhicule de transmission du savoir de la tradition, mais aussi un élément de construction et d'affirmation identitaire pour la conteuse/auteure. Pour ce faire, la confrontation des formules d'ouverture et de clôture du conte et d'autres éléments paratextuels, lieux par excellence de la simulation du narrateur, permettra de démontrer que, pour les conteuses exilées, le rôle impersonnel et simulé du narrateur traditionnel change au profit de la confidence personnelle et autobiographique.

\section{Le conte oral traditionnel}

Comme tout genre écrit, chaque genre oral a aussi ses caractéristiques spécifiques qui le différencient des autres genres de l'oraliture. Contrairement à la lodyans, unique dans son haitïanité, le conte haïtien est souvent confondu avec son équivalent européen, dont il se distingue nettement. En premier lieu, le conte haitien traditionnel fait exclusivement partie de la tradition orale, sa narration ne suppose aucun support écrit et elle s'est toujours préservée, depuis l'époque esclavagiste, à travers la transmission d'une bouche à une autre. Le contexte de 
transmission est très semblable à celui du conte de l'ouest africain, dont il descend : il est généralement proféré le soir, en plein air, sur une place ou une cour, devant un groupe de personnes, enfants et adultes rassemblées autour d'un conteur ou une conteuse, prêtes à boire sa Parole. En second lieu, tout le monde peut tirer un conte, narrer une histoire, même les enfants, comme le souligne Alex-Louise TESSONNEAU (2002 : 70-71):

Il n'existe pas de conteurs professionnels et tout participant à une veillée, (hommes, femmes, enfants), peut prendre la parole. Cependant, certains de ceux qui interviennent sont réputés car ils ont un répertoire intarissable et sont de véritables acteurs pouvant entrer dans la peau des divers personnages du conte, du récit, avec la mimique et la phonie adéquates.

Le public accorde la même importance à chaque conteur, peu importent son âge, son sexe ou sa position sociale, même s'il n'a pas les compétences techniques des conteurs professionnels ${ }^{4}$, car ce qui compte est la vérité du récit et comment elle est véhiculée. La manière dont le récit est transmis est, en effet, la troisième particularité du genre. Il s'agit d'un échange interactif intense entre le conteur et son public, dont la participation pendant la narration est fondamentale, car elle détermine la réussite de la performance. L'auditoire peut intervenir de diverses façons : chanter la chanson-guide, jouer certains personnages secondaires, mais aussi corriger le conteur en lui adressant la formule terrible et péremptoire Mèt kontè se manti! (littéralement : le conteur a menti) avec laquelle non seulement on corrige, mais on remplace le narrateur. Cependant, la substitution advient seulement en cas d'hérésie, c'est-à-dire quand une vérité primordiale est mise en question. Le but est d'inclure toutes les personnes présentes à l'événement, de n'exclure personne, d'assurer la communication et la passation, car au cours du tirage «chacun est à même d'être celui par qui passe le 'verbe' [...] chacun peut se rendre compte qu'il fait partie de l'édifice, qu'il peut apporter quelque chose, qu'il est un membre actif et qu'il participe à la vie de la communauté » (TeSSONNEAU, 2002: 71). Cette ambiance de sacralité renvoie au caractère proprement rituel du conte, qui peut être considéré un véritable rite de transmission de la parole-vérité de la mémoire collective à l'assemblée réunie autour du conteur. Comme l'explique Maximilien LAROCHE (in BARTHELEMY, $2004: 17)$ :

Le narrateur dit toujours $Y o d i$ : on m'a dit, n'étant que le porte-parole d'un yo super-narrateur qui vient d'un autre espace. Entre le narrateur transmetteur et le public il y a une coopération en vue de déchiffrer le message qui vient d'ailleurs, de loin, d'un autre espace.

Le fait d'être constamment au milieu du processus de transmission comporte l'absence de toute propriété intellectuelle, vu que le narrateur n'est que le médiateur entre la Parole ancestrale et le public présent. Ce processus s'ouvre par une formule rituelle très simple : - Krik! [conteur] // - Krak! [assistance] ; Ou encore, selon la région : - Tim, Tim ! [conteur] // - Bwa! [assistance]. Selon Alex

\footnotetext{
${ }^{4}$ Pour approfondir la différence entre conteur amateur et conteur professionnel (Maitt kont ou Mentor) voir CORINUS (2004).
} 
Louise Tessonneau, la première formule rappelle quelque chose qui se rompt, qui se détache, dans ce sens la narration du conte correspond à la donation aux autres de quelque chose qui nous appartient. La deuxième formule, reproduit par le Tim le son de la cloche, qui impose le silence, et la réponse $B w a$ (littéralement : bu) indique que l'auditoire est prêt à boire la Parole, à l'assimiler. En tout cas, dans ces formules il n'y a aucune présence du moi de l'auteur, il s'agit d'un moyen pour captiver l'attention du public, pour réveiller son intérêt. Ensuite, si la narration se passe bien, sans interruption et avec une grande participation, le conte se termine par une formule finale, comme celles-ci :-Se sa mwen t' al wè, yo ban mwen yon ti kout pye, yo voye mwen tonbe jouk isit rakonte nou sa. (C'est ce à quoi j'assistais, quand d'un coup de pied on m'a projeté jusqu'ici pour vous raconter ce que j'ai vu.) ; ou, simplement : - J'étais là et j'ai tout vu!

Ces formules sont ensuite suivies par une invitation à répandre et à semer l'histoire écoutée, afin de poursuivre la tradition de la transmission. Outre la fonction de diffusion, ce qui nous intéresse c'est comment le narrateur, à la fin de la narration, assume le rôle de témoin des aventures narrées à travers la dissimulation. En effet, non seulement il simule sa présence pour renforcer l'effet de véridicité de l'histoire, mais au lieu d'être un simple mensonge officieux pour les enfants, l'on assiste à une prise de position, l'affirmation d'être là en tant que porte-parole de la vérité. Comme l'explique Maximilien LAROCHE (2005 : 234) :

\begin{abstract}
Dans la formule de conclusion du kont, il y a une double référence à des voix collectives. Mwen, yo, nou. Mwen, c'est à la fois je et moi qui parlent. Yo, c'est eux qui me délèguent. Et nou, c'est nous tous, moi compris, mais c'est aussi, en créole haïtien, vous autres à qui je m'adresse. Il y a donc référence du discours à eux, un collectif, et adresse de ce discours à un autre collectif : nous. À noter que mwen faisant la liaison entre ces deux collectifs tout en faisant partie de chacun d'eux la communication plutôt que bipolaire serait circulaire. Il s'agirait de traiter une information, de la disséminer dans un grand ensemble bien plus que de la faire passer d'un émetteur à un récepteur fortement différenciés sinon même opposés.
\end{abstract}

À travers une simple formule de clôture, le conteur-narrateur consolide sa parole et, en même temps, sa position de médiateur entre un supernarrateur, le yo, lointain et ancestral, et le public-narrataire proche et présent. De plus, à l'intérieur de la narration il serait impossible de repérer des traces autobiographiques du conteur/narrateur, car il ou elle est seulement un intermédiaire. Or, dans le passage à la forme écrite l'on assiste à plusieurs changements concernant la langue et le style de la narration, mais aussi la position du narrateur. En effet, si déjà à partir des recueils ethnographiques des contes de la tradition, l'on pourrait se questionner sur la limite subtile entre transcription et réécriture ${ }^{5}$, dans le conte d'auteur il s'agit d'une pleine propriété intellectuelle.

\footnotetext{
${ }^{5}$ Pour approfondir voir DeL Rossi (2014).
} 


\section{Le conte defolklorisé de Paula Clermont Péan}

Le recueil Chaque Malice a son Bouqui / Chak Malis gen Bouki pa l (2009), de la conteuse haïtienne Paula Clermont Péan, est l'un des premiers recueils d'auteur publiés en Haïti. Cet ouvrage permet de comprendre les modifications apportées aux techniques narratives de la simulation dans le passage de la version orale à celle écrite. Le premier élément à souligner est qu'il s'agit d'une œuvre littéraire, ce qui exclut toute appartenance au cadre ethnographique. En effet, Paula Clermont Péan n'est pas seulement une conteuse professionnelle, mais aussi actrice et dramaturge. Son appartenance à l'art dramatique influence énormément sa manière de s'approprier les contes de la tradition, qui sont souvent réadaptés pour la mise en scène. Un autre facteur fondamental pour comprendre les choix stylistiques de la conteuse est son côté philanthropique, en tant que directrice du Centre Culturel Pyepoudre ${ }^{6}$, dont le but est de promouvoir la culture haïtienne chez les jeunes à travers l'approche théâtrale. La volonté de soutenir et diffuser le patrimoine oral haïtien à l'intérieur et en dehors du Pays se retrouve avant tout dans le choix du sujet narré : le cycle de Malice et Bouqui. Il s'avère que ces deux personnages sont les plus célèbres du folklore de l'île et qu'ils représentent parfaitement l'esprit haïtien, comme l'affirme Emmanuel PAUL (1962 : 14) :

Du point de vue de la diffusion interne, à part la catégorie Bouki et Malice, on ignore laquelle des autres est plus répandue. [...] Malice résume le style de notre peuple qui le reproduit sans cesse à des milliers et des milliers d'exemplaires. [...] Malice symbolise donc notre type national en ce qu'il a de plus caractéristique dans la mesure que ces traits forment notre personnalité de base.

Un autre élément qui souligne l'intention de la conteuse de semer sa parole audelà des frontières nationales est la façon assez particulière de la narratrice de s'adresser au lecteur. L'ouvrage est destiné, effectivement, autant au lectorat haïtien qu'au lectorat francophone, non seulement pour le choix de la version bilingue français/créole, mais surtout par la technique narrative choisie, un mélange entre la narration haïtienne et européenne. Le recueil s'ouvre par une introduction qui présente l'origine des contes choisis :

Cette histoire remonte à la nuit des temps. Elle existe depuis le temps où ti konkonm $t$ ap goumen ak berejèn [en créole dans le texte, « depuis des temps immémoriaux », littéralement : «depuis que les petits concombres luttent/se battent avec les aubergines »]. Je l'ai apprise sur les genoux de Tonton Olicha. Elle lui était arrivée par delà les frontières, la légende de Bouqui et Malice. Elle avait parcouru beaucoup de pays, d'océans, de villes, de villages et sentiers, de l'Orient à l'Occident, du Nil à la Caraïbe. Cette histoire a peut-être perdu des plumes; les personnages et les lieux ont peut-être changé, mais chez nous, elle a pris beaucoup d'ampleur, de couleur et de vie. Ici, on ne saurait se passer de nos deux héros, Bouqui et Malice, qui vivent de multiples aventures, chaque jour qui vient. Malice en sort toujours gagnant et Bouqui, lui, semble être né pour se faire avoir. Frère, cousin, oncle, neveu, ami, ennemi, ils ne peuvent pas vivre séparés l'un de l'autre. Bouqui et Malice sont liés comme frères siamois. C'est pour cela que je dis : «Chaque Malice a son Bouqui ». Alors, prends ta place dans le cercle! Prends ta

\footnotetext{
${ }^{6}$ Pour plus d'informations voir la page internet du Centre $:$ http://www.pyepoudre.org/about.
} 
petite chaise! Que tes yeux et surtout tes oreilles soient bien ouverts! Je vais te raconter. (CLERMONT PÉAN, 2009 : 6)

Cette introduction contient deux processus qui s'opposent: le premier est la recréation du contexte traditionnel de la transmission de la parole-vérité du conte, par l'historique des récits. En effet, non seulement ils remontent à la «nuit des temps », mais ils ont traversé les «frontières » jusqu'à arriver à son oncle, qui les lui a transmis. De plus, la narratrice établit le contexte caribéen à travers des expressions comme «chez nous », « ici », « on ne saurait», mais elle recrée aussi l'ambiance d'une collectivité avec la référence au «cercle » typique du tirage des contes. De l'autre côté, l'on entrevoit une attitude condescendante envers le lecteur non créolophone dans la description des deux protagonistes du cycle, mais aussi dans le manque des formules traditionnelles d'ouverture, qui pourraient heurter le lectorat inexpert. Aucun Krik, aucun Krak, la conteuse va simplement « raconter », elle ne demande aucune réponse, aucun échange avec son lectorat; le but originaire du conte, la transmission, semblerait ainsi être perdu. D'ailleurs, la narratrice s'attend à un public attentif, aux yeux et aux oreilles «bien ouverts", assis sur de petites chaises, mais muet. Cet esprit de tendresse et d'adaptation à l'auditoire est amplifié par le remplacement des formules traditionnelles d'ouverture par de petites introductions naïves et rêveuses, qui accompagnent doucement le lecteur à travers les images et les aventures exotiques narrées. Voici quelques exemples : «Tout comme le vent vous apporte des feuilles d'automne jaunies par la saison, mon conte vous apporte le mystère et le rêve. » (CLERMONT PÉAN, 2009: 24); ou encore: "Aujourd'hui, mon conte, ce bout d'histoire est une légende pleine d'images qui flottent dans ma tête mais je n'arrive pas à lui trouver un nom. Je sais seulement que je chante sur les collines d'ombre de l'aurore. » (CLERMONT PÉAN, 2009 : 48).

Nous sommes loin du style du conte haïtien traditionnel, les formules rituelles se transforment en descriptions poétiques, qui heurtent parfois l'imaginaire caribéen, comme par exemple «les feuilles d'automne jaunies» qui contrastent avec la verdure luxuriante d'Haïti. Pourtant, ces préludes conservent au moins l'esprit de la transmission, surtout grâce au topos traditionnel du Vent de la Caraïbe $^{7}$, qui sème le Savoir et la Parole. Enfin, la volonté de diffusion se retrouve aussi dans l'épilogue du recueil :

Voilà, j'ai confié mon conte à vos mémoires, mais vous ne pouvez le garder pour vous. Sinon il sera vite oublié, jeté dans la poubelle de l'oubli. Prenez mon conte, ce rêve, ce mystère, laisse-le murir en vous et confiez-le au vent du soir. Faites-le parcourir d'autres sentiers, courir d'autres horizons... (CLERMONT PÉAN, 2009 : 56)

\footnotetext{
${ }^{7}$ Le Vieux Vent Caraïbe, personnage mythique du folklore haïtien et antillais, est la représentation de l'art du conte et de son pourvoir de transmission. Célèbre est la représentation donnée par l'écrivain Jacques Stephen ALEXIs (2008: 11) dans son ouvrage Romancero aux étoiles : «Ainsi, j'avais devant moi mon aïeul, le Vieux Vent Caraïbe, l'immémorial et légendaire ménétrier, le plus grand Samba de toute la Caraïbe. Depuis un temps que nul ne peut dire ce vieux drille chante, danse, musarde, se musse, musique et fredonne au long des âges nos belles histoires de jadis, de naguère et de toujours. »
} 
Voici donc retrouvée la tradition : la conteuse fait appel à son auditoire (hélas muet) dans une communication simulée, mais à voie unique. C'est la technique du passeur de Parole, le mwen, le yo et le nou reviennent à la surface. En effet, tout comme dans le conte oral traditionnel, la voix narrative est anonyme dans son rapport avec le lecteur, même si elle donne sa version du conte, même si le «je »a confié son conte. Mais qui est ce «je»? Plusieurs traces révèlent qu'elle est une femme haïtienne, comme le montre cet extrait :

Aujourd'hui, je suis encore hantée par cette histoire qui vit dans notre imaginaire collectif, vieille de je ne sais combien de temps. Je suis tentée de commencer par: "Il y a quelques temps, lorsque régnait la paix dans notre pays"...ou bien : «Il y a longtemps de cela, quand le pays était prospère »... ou : « Un jour lointain, quand le rêve avait une place dans la vie des hommes »... quand... quand... quand... (CLERMONT PÉAN, 2009 : 32)

Pourtant, la narratrice semblerait plutôt inexpérimentée : elle doute souvent de la gestion de sa narration, ou plutôt de la gestion de ce nouveau public occidental, qui ignore le créole, les proverbes, les personnages. D'une part l'on assiste à la tentative de respecter les règles traditionnelles du tirage, mais l'écart entre les deux publics est évident, tout comme les démarches pour s'adapter à cette nouvelle condition. Certes, c'est en vérité l'auteure qui fait face au passage de l'oral à l'écrit, du créole au français, cette question éternelle qui hante chaque écrivain haïtien. Cependant, nous ne pouvons pas établir une correspondance parfaite entre la narratrice et l'auteure, Paula Clermont Péan, car l'on relève le manque de toute référence à sa personne, une dissimulation de sa propriété intellectuelle. Elle maintient donc une distance entre sa vie privée et son rôle de conteuse, entre sa biographie et son lectorat, une distance qui n'existe pas dans le conte en contexte diasporique.

\section{Mimi Barthélémy : entre pédagogie et témoignage personnel}

Mimi Barthélémy, née à Port-au-Prince en 1939, arrive en France à l'âge de seize ans pour continuer ses études et, après une vie assez aventureuse qui l'amène à faire le tour du monde, elle s'établit à Paris, plus précisément dans le quartier de Château Rouge, près de la Goutte d'Or, où elle recrée un petit coin de la Caraïbe et où elle reste jusqu'à sa mort, survenue en 2013. Avant d'aborder l'art du conte en 1983, elle avait déjà une carrière de comédienne et de metteuse en scène, qui influence énormément son approche à l'oraliture haïtienne, comme elle en témoigne elle-même :

On dit que l'art du conte est un art du détour. Eh bien, curieusement, c'est par le détour du théâtre que je me suis mise à conter. Je commençai par théâtraliser mes contes que je jouais dans des réseaux de théâtre. Je resterai toujours marquée par ces débuts, ainsi que par les influences qu'a eues sur ma formation le théâtre militant, en étant une conteuse de scène habitée par la passion de son île et célébrant son identité menacée et retrouvée. Lorsque rapidement je cesserai de théâtraliser mes histoires pour devenir une conteuse à voix nue, je me définirai toujours comme une conteuse de scène ; mon goût immodéré pour les planches en est en grande partie la cause. (BARTHELEMY, 2008) 
À cette influence des planches s'ajoute aussi un triple engagement actif de la comédienne-conteuse: pour la transmission de la mémoire d'Haïti, pour la transmission du patrimoine oral haïtien et, enfin, pour la défense des langues françaises, le français haïtien et le français de France. Cet engagement comporte une transmission des contes assez traditionnelle, qui respecte souvent les formules de clôture et fermeture. Une autre particularité est la volonté de ne pas concevoir le conte comme un genre uniquement pour la jeunesse, mais aussi pour un public adulte. Cette double visée comporte deux différentes façons de s'adresser au lecteur. La première est bien visible dans l'ouvrage Haïti conté (2004), une anthologie de contes qu'elle a répertoriés et remaniés, mais c'est surtout une guide pour apprendre à conter et s'approcher de la culture haïtienne. L'ouvrage présente, en effet, un long avant-propos où l'auteure offre en trente pages le portrait de son pays d'origine, du patrimoine artistique et culturel, mais aussi des aspects sociologiques de la vie quotidienne. L'anthologie est divisée en sections, chacune consacrée à un sujet et ouverte par des formules traditionnelles ou par des citations littéraires. Enfin, dans la partie conclusive, les annexes, par lesquels la conteuse conclut son cours magistral de tirage de conte, donnent au lecteur-élève des conseils pratiques pour choisir le conte par rapport à l'âge des spectateurs. La démarche choisie permet à Mimi Barthélémy non seulement de respecter le rituel traditionnel de la narration, avec ses formules, l'anonymat narratif et la simulation des événements qui concernent le narrateur, mais aussi de recouper à l'intérieur de l'ouvrage une partie où la conteuse, bannissant toute simulation, développe et exploite le côté pédagogique du conte.

L'autre type de lecteur auquel l'auteure s'adresse est l'enfant, dans le cadre de la littérature de jeunesse. Les changements d'une version à l'autre, selon l'âge visé par le recueil, sont bien évidents, non seulement du point de vue de la mise en page, mais, en général, en fonction de l'édition dans le choix de la version audiolivre.

Une autre différence fondamentale concerne la modalité narrative. En effet, toujours dans le respect de son but de diffusion de la culture orale haïtienne, les recueils pour les enfants observent, souvent avec respect, les rituels de la narration. Par exemple, dans Contes d'Haïti (2011) les contes s'ouvrent et se terminent par des formules traditionnelles, comme la suivante :

Lorsque j'ai demandé à Bouki s'il connaissait le proverbe «Les œufs ne doivent pas se glisser dans la ronde des pierres », cela lui a rappelé ses mésaventures avec Tibèf et l'a mis hors de lui ; il m'a donné un coup de pied qui m'a projetée de là-bas jusqu'ici, juste à temps pour vous raconter cette histoire. (BARTHÉLÉMY, $2011: 13$ )

Comme dans les contes de Paula Clermont Péan, ici aussi le je reste anonyme, sans aucune référence à la conteuse en chair et os, en même temps la narratrice simule sa présence, alors que l'auteure s'éclipse derrière un effet de dissimulation .

Encore plus curieuse est la confrontation avec le recueil de Paula Clermont Péan si l'on analyse l'ouverture du recueil :

Sous mon avocatier j'ai trouvé sept contes que j'aimerais vous faire déguster, selon le menu que j'ai concocté. [...] Ceux du milieu sont un plat populaire de grillot de cochon et de bananes 
plantains frites, agrémenté d'une salade épicée à la sauce ti malice. Cette sauce tire son nom d'un personnage populaire de la tradition orale haïtienne, Malice, le roi de la ruse. Son partenaire, Bouki, lui, est le roi de la sottise. (BARTHÉLÉMY, $2011: 4$ )

La narratrice fait semblant d'avoir trouvé les contes « sous son avocatier » et de les avoir concoctés, c'est-à-dire remaniés et réécrits, selon sa recette personnelle. Dans le court extrait l'on dénote plusieurs éléments qui caractérisent la narratrice en tant que Haïtienne, en particulier la présence des plats typiques de la gastronomie haïtienne. Comme dans le recueil de Paula Clermont Péan, le ton et le style s'adressent de toute évidence au jeune public français, qui est initié à l'univers caribéen avec sa verdure, ses fruits et ses plats savoureux. Mimi Barthélémy semble avoir trouvé le juste équilibre dans sa narration, qui ne transgresse pas trop les règles de la tradition, mais en même temps s'adapte au contexte diasporique.

Néanmoins, dans le recueil La Reine des Poissons (2014) la fidélité à la tradition trouve son exception. La Reine des Poissons est l'un des premiers contes adaptés par la conteuse, elle l'a publié plusieurs fois, chaque fois dans une nouvelle version :

Lorsque je suis devenue conteuse j'ai trouvé une version de la reine des poissons, écrite par Philippe Thoby Marcelin et Pierre Marcelin, dans un recueil de Contes et Légendes d'Haïti publié chez Nathan en 1967, sur laquelle j'ai travaillé. [...] Je publierai une première version de ce conte sous le titre La Femme Thazar. La deuxième version, publiée aux Éditions Vif Argent, dans la collection cassettine, s'appellera La Reine des Poissons. Je reprendrai La Femme Thazar dans mon recueil de contes d'Haïti, publié aux éditions Slatkine en 2004, Haïti Conté. (BARTHÉLÉMY, $2011: 33$ )

En 2010, Mimi Barthélémy commence à travailler à une nouvelle version, qui sera publiée à titre posthume seulement en 2014. Dans ce dernier projet, l'on dénote certains changements par rapport aux autres recueils. Le conte s'ouvre et se conclut par une chanson-guide, comme dans la tradition des contes chantés, mais la narration débute de façon assez particulière : «Il était une fois, il y a longtemps de cela, en Haïti, dans mon île, un pêcheur nommé Lormilis » (BARTHÉLÉMY, 2011 : 1). Ce début ne représente pas la formule traditionnelle d'ouverture, au contraire il s'ouvre par la formule très européenne « Il était une fois », mais tout de suite la narratrice affirme son identité haïtienne, non seulement en nommant Haïti, mais en soulignant son appartenance avec la phrase «dans mon île ». Cependant, la véritable particularité de cet ouvrage réside dans le péritexte : en effet à la fin, après la traduction en créole du conte, l'ouvrage offre en annexe un court texte autobiographique qui a pour titre Pourquoi je raconte La reine des poissons. Sur ces pages le je abandonne toute forme de simulation traditionnelle, pour raconter à la première personne son histoire personnelle et ses liens intimes avec le conte qu'elle vient de narrer :

La reine des poissons est l'un des rares contes qui me restent de mon enfance. J'ai en mémoire, encore aujourd'hui, l'image à la fois angoissante et émouvante du pêcheur attiré par la reine des poissons, qui la rejoint au fond de la mer. Je me rappelle la voix aiguë de la conteuse, à ce moment-là ; elle chantait avec sa « voix conte ». Mais je n'ai plus souvenir de la mélodie; il me reste seulement un sentiment de ravissement et un petit pincement au cœur. Je me souviens que 
nous écoutions ce conte, assis sur le bord de l'énorme bassin qui surplombait la propriété. [...] J'en ai fait mon logo durant plusieurs années. Le temps a passé et même lorsque j'ai cessé de la conter, pendant quelque temps, elle, la reine des poissons, m'est toujours restée collée à la peau. Je vis en France, grâce à elle, dans mes eaux territoriales. (BARTHÉLÉMY, $2011: 35$ )

Il s'agit d'une véritable mini-autobiographie de quelques pages, le je se répète constamment et les verbes du souvenir s'enchaînent : «J'ai en mémoire », «il me reste seulement», «je me souviens», «je n'ai plus souvenir». Dans son témoignage, la conteuse parcourt en trois pages les étapes fondamentales de sa vie, en les liant au conte La reine des poissons et au mythe de la Femme-thazar, protagoniste du récit. Ainsi l'identification finale entre la conteuse et le conte se réalise dans le logo, sorte de talisman ou fétiche qui la protège hors de sa patrie natale. Dans plusieurs entretiens ${ }^{8}$, Mimi Barthélémy confesse avoir retrouvé sa voix intérieure à travers la pratique des contes et souligne la fonction thérapeutique que le conte a jouée dans sa réaffirmation identitaire en France. La Reine des Poissons est tellement important pour la conteuse, qu'elle rompt la règle de la distanciation dissimulatrice, en se laissant emporter par le récit autobiographique, touchée dans son intimité par son rapport exclusif avec ce récit. En tout cas, même si l'on entrevoit déjà cette volonté de liberté au début du conte, dans une première et subtile affirmation identitaire, le péritexte devient le véritable espace de liberté pour satisfaire le besoin du témoignage, en dehors de toute forme de (dis)simulation.

\section{L'aventure autobiographique dans le conte de Joujou Turenne}

Si Mimi Barthélémy pose une distinction entre vérité et fiction, dans les contes de Joujou Turenne, conteuse haïtienne-québécoise, l'on observe une intromission continuelle de la réalité dans la fiction. En effet, la simulation typique de l'espace fictionnel entre en contraste avec le témoignage personnel, qui s'insère à plusieurs reprises dans le texte.

Née en Haïti, au Cap, encore très jeune Joujou Turenne est contrainte à suivre sa famille en exil au Québec à cause de la dictature de François Duvalier (19571971) et de son fils Jean-Claude (1971-1986). Dans sa nouvelle terre d'accueil multiculturelle elle fait ses études et obtient une spécialisation en danse thérapeutique. Ensuite, à cette première passion pour la danse s'ajoutera celle pour l'art dramatique. Seulement après plusieurs années, vers 1990, elle découvre sa vocation de conteuse. Un lecteur attentif pourrait connaitre la vie et le parcours artistique de Joujou Turenne juste en lisant ses recueils, comme le montre l'extrait suivant, tiré de son premier recueil, Joujou, Amie du Vent :

Quand j'étais petite, je me souviens que par des nuits étoilées je prenais un plaisir fou à me faire raconter des histoires. Des histoires à faire rire, pleurer ou encore frémir de peur. Mais mon enfance s'est brusquement arrêtée dans une valise. Et mes souvenirs se sont posés sur les ailes d'un gros oiseau pour s'envoler vers des nuages nommés EXIL. Avec l'exil je suis tombée dans l'oubli je suis tombée dans l'ennui. Et pour oublier l'oubli et me désennuyer de l'ennui j'ai

\footnotetext{
${ }^{8}$ Voir par exemple Mimi Barthélémy, Naissance d'une vocation (BARTHELEMy, 2008).
} 
dansé. [...] Puis j'ai arrêté de danser. J'ai arrêté de danser car la danse à elle seule n'arrivait pas à être le reflet de mon âme. Je me suis donc rendue au carrefour du réel et de l'imaginaire. Làbas, en ces lieux insolites j'ai rencontré des personnages merveilleux et leurs histoires. [...] Et me voici, me voilà, messieurs, dames, société, depuis lors prête à livrer mon histoire, mes histoires à tout vent à quiconque veut bien écouter. KRIK! KRAK ! (TURENNE, 1999 : 6)

Déjà à partir de la première phrase du recueil, la conteuse nous plonge dans son histoire. Même si à une première lecture sa narration pourrait paraître une simulation traditionnelle, il suffit d'avancer dans la lecture pour se heurter avec la réalité la plus frappante et la plus douloureuse: l' «EXIL ». À partir de ce moment, la narration se transforme de plus en plus en une autobiographie fragmentaire, les frontières entre vérité et fiction s'effacent en brouillant le lecteur. Selon JeanPhilippe MiRAUX (2009 [1996] : 11-12), l'autobiographie est le moyen de la reconquête de soi, de la reconstruction et de la reconstitution de son propre être. De ce fait, la narration choisie dans l'introduction souligne sa volonté d'affirmer son identité de conteuse après le traumatisme de l'exil. Effectivement, si l'on considère les caractéristiques du style de l'autobiographie proposées par Jean STAROBINSKI (in MIRAUX, 2009 : 15-16), l'on notera que ce bref extrait respecte toutes les conditions :

1) la présence d'une identité du narrateur et du héros de la narration ;

2) la prévalence de la narration sur la description ;

3) la présence de la notion de parcours ou de tracé d'une vie.

L'on retrouvera aisément chaque condition à l'intérieur de la narration de Joujou Turenne; pourtant, il ne faut pas oublier qu'il s'agit d'un recueil pour la jeunesse, par conséquent les expériences autobiographiques sont recouvertes d'une patine de merveilleux. Pourtant, le but est bien clair: Joujou Turenne veut livrer son histoire dans ses histoires. Au cours du recueil les interventions et les formules typiques du conteur haïtien rythment la narration, comme dans une vraie veillée de tirage de conte, grâce aussi aux effets typographiques qui rendent l'oralité. Cependant, les interventions traditionnelles sont interrompues, ou mieux, alternent avec des flux de conscience de la conteuse, en particulier le traumatisme de l'exil :

EXIL, QU'AS-TU FAIT À MA MEMOIRE ? Heureusement que j'ai de très bons amis qui me nourrissent l'âme. Car voyez-vous quand on est l'amie du Vent, l'amie des fées, l'amie du Rêve, de la Parole et de la Mer, il nous arrive de ces choses incroyables. (TuRENNE, 1999 : 84)

La caractéristique de ce type de narration est donc le mélange harmonieux entre la confession autobiographique et les effets de simulation, comme dans l'extrait mentionné, qui s'ouvre par la question qui hante la conteuse exilée et sa ré-pacification avec elle-même grâce au conte et à son pouvoir merveilleux. Le conte est donc pour Joujou Turenne sa manière de construction et de reconstruction identitaire, comme nous pouvons le noter dans ce dernier extrait, clôture du recueil :

Et moi, Joujou, amie du Vent

Fille des îles

Fille d'exil

née sur une île 


\section{Haïti}

vivant sur une autre île

Montréal

j'ai partagé avec vous une partie de mon île intérieure

S'il vous prend l'envie de raconter toute mon aventure, par bribes ou dans son entièreté, je n'en tire aucune offense. Ces paroles avant d'être les miennes appartenaient au Vent, à la Mer, aux fées, au Temps, au Rêve et à la Parole elle-même. À présent, elles vous appartiennent... Semezles à tout vent et à quiconque voudra bien écouter. (TURENNE, $1999: 158$ )

Chez Joujou Turenne, l'effet thérapeutique du conte est évident, car à l'invitation traditionnelle à partager les contes s'ajoute une prise de conscience identitaire, qui résulte de l'harmonisation des deux univers de la conteuse, car tout comme dans ses contes, elle fait fusionner la tradition et la modernité. Aucun effet de dissimulation : dans cet épilogue l'auteure et la narratrice se superposent grâce à un équilibre parfait entre vérité et fiction, où le public non seulement partagera les récits de la tradition, mais aussi «l'aventure » biographique de la conteuse.

\section{Conclusion}

Pour conclure, le conte haïtien contemporain n'est plus seulement un moyen de transmission de la mémoire collective, mais il devient un moyen de réaffirmation identitaire, un réservoir de mémoire identitaire collective et individuelle à la fois. L'approche comparative privilégiée dans cette étude a cherché à démontrer la variation de la figure du narrateur à l'intérieur du conte, soit du point de vue chronologique (conte traditionnel Vs. conte contemporain), diatopique (conte haïtien Vs. conte haïtien en diaspora) et du passage de l'oral à l'écrit. En effet, d'un premier état de simulateur anonyme, caractéristique du narrateur du conte oral traditionnel, l'on passe à trois types différents de narration. Le premier, celui de Paula Clermont Péan, est caractérisé par une demi-fidélité aux règles traditionnelles de la narration, vu que la narratrice utilise seulement les formules de clôture, en évitant les formes folkloriques d'ouverture pour ne pas bouleverser un public non haïtien. Par contre, Mimi Barthélémy, d'une part préserve le style traditionnel dans le but pédagogique de diffuser les techniques primordiales et, parallèlement, se ménage un espace à soi à l'intérieur de l'ouvrage, consacré au témoignage personnel de sa renaissance loin de sa patrie. Le dernier cas analysé, celui de Joujou Turenne, bouleverse les règles traditionnelles de la narration, grâce à une superposition totale de la narratrice et de l'auteure, en dehors de toute technique de (dis)simulation. Cette rupture est liée à la transformation du genre, dont le but, en contexte diasporique, n'est plus seulement d'éduquer en amusant, mais aussi de témoigner des expériences individuelles. Les changements sociaux entraînés par la diaspora ont donc changé radicalement le conte, en lui confiant la tâche de transmission de la mémoire collective et personnelle. L'évolution se résume dans la figure du narrateur, qui doit se bâtir une identité bien précise, un je qui n'est plus seulement (dis)simulateur, mais passeur de vérité universelle et personnelle. 


\section{BIBLIOGRAPHIE}

AlEXIS Jacques Stephen (2008), Romancero aux étoiles, Paris, Gallimard ; $1^{\text {ère }}$ édition 1960, Paris, Gallimard.

ANGLADE Georges (1999), Les Blancs de Mémoire, Montréal, Boréal.

BARTHELEMY Mimi (2008), Mimi Barthélémy. Naissance d'une vocation http://ile-en-ile.org/mimi-barthelemy-naissance-dune-vocation/

BARTHÉLÉMY Mimi (2004), Haïti conté, Genève, Slatkine.

BARTHELEMY Mimi (2011), Contes d'Haïti, Toulouse, Milan.

Barthelemy Mimi (2014), La Reine des Poissons, Paris, Kanjil.

Clermont PeAn Paula (2001), Le chant de Miraya, Port-au-Prince, Editions Mémoire.

Clermont PeAn Paula (2009), Chaque Malice a son Bouqui/Chak Malis gen Bouki pa l, Coconut Creek, Educa Vision Inc.

CORINUS Véronique (2004), Les contes créoles: état des lieux, Contes et Romans - Univers Créoles 4, Paris, Anthropos.

DeL Rossi Sara (2014), Le Roman de Bouqui, entre tradition et innovation, InterFrancophonies (version en ligne) http://interfrancophonies.org/images/pdf/melanges/delrossi_2014.pdf

LAROCHE Maximilien (1991), La double scène de la représentation. Oraliture et littérature dans la Caraibe, Québec, GRELCA.

LAROCHE Maximilien (2000), De l'Oraliture à la Littérature, in : Découvrir Haïti àtravers ses écrivains (Actes $\mathrm{du}$ colloque) http://www.easyasbl.be/uploads/6/2/1/6/62169429/ecrivains-conferencemaximilien-laroche-oraliture.pdf

LAROCHE Maximilien (1998), Le non-dit du discours haïtien, in : CHEVRIER Jacques (1999), Poétiques d'Edouard Glissant. Actes du colloque «Poétiques d'Edouard Glissant »Paris-Sorbonne, 11-13 mars 1998, Paris, Presses de l'Université Paris-Sorbonne, p. 233-243.

LEVY Joseph (2004), L'espace d'une génération: entretien avec Georges Anglade, Montréal, Liber.

MIRAUX Jean-Philippe (2009), L'autobiographie. Écriture de soi et sincérité, Paris, Armand Colin ; 1 ère édition 1996, Paris, Nathan.

PAUL Emmanuel (1962), Panorama du Folklore Haïtien, Port-au-Prince, Imprimerie de l'État.

TESSONNEAU Alex-Louise (2002), Le «Kont» créole : à l'interface de l'écrit et de l'oral, Etudes Créoles XXV n², Paris, L'Harmattan.

Tsoungui Françoise (1986), Clés pour le conte africain et créole, Paris, CILF, Edicelf.

TURENNE Joujou (1999), Joujou Amie du Vent, Montréal, CIDHCA. 